\title{
Theoretical models for describing longitudinal bunch compression in the neutralized drift compression experiment
}

\author{
Adam B. Sefkow and Ronald C. Davidson \\ Plasma Physics Laboratory, Princeton University, Princeton, New Jersey 08543, USA
}

(Received 7 June 2006; published 5 September 2006)

\begin{abstract}
Heavy ion drivers for warm dense matter and heavy ion fusion applications use intense charge bunches which must undergo transverse and longitudinal compression in order to meet the requisite high current densities and short pulse durations desired at the target. The neutralized drift compression experiment (NDCX) at the Lawrence Berkeley National Laboratory is used to study the longitudinal neutralized drift compression of a space-charge-dominated ion beam, which occurs due to an imposed longitudinal velocity tilt and subsequent neutralization of the beam's space charge by background plasma. Reduced theoretical models have been used in order to describe the realistic propagation of an intense charge bunch through the NDCX device. A warm-fluid model is presented as a tractable computational tool for investigating the nonideal effects associated with the experimental acceleration gap geometry and voltage waveform of the induction module, which acts as a means to pulse shape both the velocity and line density profiles. Self-similar drift compression solutions can be realized in order to transversely focus the entire charge bunch to the same focal plane in upcoming simultaneous transverse and longitudinal focusing experiments. A kinetic formalism based on the Vlasov equation has been employed in order to show that the peaks in the experimental current profiles are a result of the fact that only the central portion of the beam contributes effectively to the main compressed pulse. Significant portions of the charge bunch reside in the nonlinearly compressing part of the ion beam because of deviations between the experimental and ideal velocity tilts. Those regions form a pedestal of current around the central peak, thereby decreasing the amount of achievable longitudinal compression and increasing the pulse durations achieved at the focal plane. A hybrid fluid-Vlasov model which retains the advantages of both the fluid and kinetic approaches has been implemented to describe the formation of pedestals in the current profiles. The comparison between the experimental measurements and the various theoretical models is excellent.
\end{abstract}

DOI: 10.1103/PhysRevSTAB.9.090101

PACS numbers: 52.59.Sa, 29.27.- a, 52.59.Fn

\section{INTRODUCTION}

The final transport section leading to the target offers a significant challenge for the development of heavy ion drivers for applications such as high energy density physics, warm dense matter, and heavy ion fusion [1-3]. Heavy ion drivers can deliver more intensity to the target per unit length of accelerator by greatly compressing intense ion charge bunches over short distances. In one example, intense ion beams [4-6] are transversely focused to final diameters less than a few $\mathrm{mm}[7,8]$ and longitudinally focused to pulse durations less than 10 ns. Maximizing such compression offers the potential of compressing heavy ion beams to very high current densities. Therefore, shorter accelerators and transport lines could be used as heavy ion drivers, making them more compact and cost effective than previously envisioned.

The majority of the ion beam's space charge must be neutralized during the final transport stage $[9,10]$ in order to overcome the defocusing self-field forces and achieve small spot size with short pulse duration. The Heavy Ion Fusion Science Virtual National Laboratory constructed the neutralized transport experiment (NTX) at the Lawrence Berkeley National Laboratory in order to study the physics of the transverse focusing and neutralized transport required for achieving small spot sizes with space-charge-dominated beams [11]. Experiments [12,13] and simulations $[14,15]$ have shown that a high-density plasma positioned "upstream" of the target chamber provides a sufficient electron population to neutralize the beam's space charge, thereby allowing the intense beams to be focused beyond the space-charge limit. The NTX injector generated a low-emittance and variable-perveance $K^{+}$ion beam with up to $400 \mathrm{keV}$ of directed energy. Located upstream of a dense background plasma, a finalfocus magnet set gave the beam a convergent angle just before the beam entered the neutralized drift region. Final spot radii of $\sim 1 \mathrm{~mm}$, corresponding to current density compression factors of approximately 200, were measured at the focal plane.

Each ion beam pulse must be compressed longitudinally in order to achieve pulse durations less than $10 \mathrm{~ns}$ at the focal plane. Longitudinal focusing is achieved by applying a time-dependent velocity tilt to an intense charge bunch and subsequently allowing it to drift through a neutralizing background plasma. The beam's pulse length compresses longitudinally due to the applied longitudinal velocity tilt [16]. This concept is referred to as "neutralized drift 
compression," and the upgrade of the NTX facility is called the neutralized drift compression experiment (NDCX).

A schematic of the NDCX experimental setup is shown in Fig. 1. The primary upgrade component from NTX to NDCX is the induction module, which is a linear induction accelerator [17] with one acceleration gap. The ferromagnetic induction core, voltage pulse modulators, and the acceleration gap, together constitute the induction module. The induction module is responsible for the impression of the axial velocity tilt on the beam pulse by means of a timedependent voltage waveform. The voltage waveform has two extrema, with a change in polarity at the halfway point, such that the first half of the beam pulse (the "head") experiences a net decelerating electric force, and the second half of the beam pulse (the "tail") experiences a net accelerating electric force. Thus, the beam pulse acquires a head-to-tail velocity tilt immediately before it enters the region containing background plasma, where the tilted beam drifts until the focal plane in current is reached. Since the current density, and therefore the relative power, of the charge bunch grows in the intermediate region of the applied tilt, where the decelerated head meets the accelerated tail, while the beam drifts through a background plasma, the phrase "neutralized drift compression" explicitly refers to this process.

The main goal of experiments on the NDCX device is to determine the experimental limits, both physical and technological, of the longitudinal current density compression. Particle-in-cell (PIC) simulations predict a pulse duration of a few ns, with up to about one $A / \mathrm{cm}^{2}$ of current density at peak compression for initial experiments [18]. Accurate experimental measurements of longitudinal compression factors of 60 times the initial beam current with pulse durations of 4 ns have been made and compare favorably to simulation $[18,19]$. Longitudinal compression of spacecharge-dominated ion beams without the aid of background neutralizing plasma has also been studied [20,21], but the associated longitudinal compression was significantly reduced relative to the neutralized case, due to the effects of ion beam space charge.

Theoretical models of experimental results obtained in the NDCX device provide valuable insights into the physi-

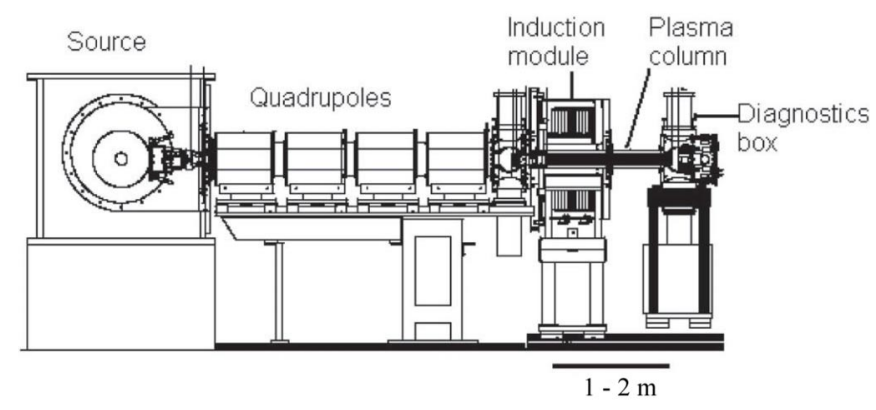

FIG. 1. Schematic of the NDCX experimental setup. cal and technological limitations of neutralized drift compression. A macroscopic warm-fluid model used to investigate a number of realistic experimental conditions found in neutralized drift compression experiments is presented in Sec. II. In Sec. III, a kinetic model based on the Vlasov equation is employed in order to numerically obtain dynamically compressing solutions for an intense charge bunch with NDCX-relevant parameters. A hybrid fluidVlasov model combines the main advantages of the warm-fluid and kinetic formalisms and is described in Sec. IV. Comparisons between the kinetic model, hybrid fluid-Vlasov model, PIC simulations, and experimental results are discussed in Sec. V. A brief summary and discussion of the numerical models are provided in Sec. VI.

\section{MACROSCOPIC FLUID MODEL}

The general challenge for theoretical models is to realistically and tractably describe the important physics in associated experiments. Substantial progress has been made on the theory of neutralized drift compression, including the development of a kinetic formalism based on the Vlasov equation [22] and macroscopic fluid models [6,23-26]. However, in order to concisely compare models with measurements, a number of experimental conditions need to be integrated into theoretical descriptions of neutralized drift compression, such as the effects of nonideal velocity tilts in beams with finite temperature, partial beam neutralization by the background plasma, the space charge associated with an imperfectly neutralized beam, finite acceleration gap size, and plasma temperature. Each of these effects can give rise to unwanted consequences such as increased pulse lengths at focus, focal plane smearing, and reduced compression factors (both in the transverse and longitudinal directions).

The technique of particle-in-cell (PIC) simulation has been the primary computational tool for self-consistently including all of the aforementioned realistic effects in one model for comparison with neutralized drift compression experiments. A PIC code [27] evolves the full distribution functions of all simulated species for given initial and boundary conditions and, therefore, is an invaluable resource for computational physicists. However, PIC simulations are generally noisy and require a large amount of computational time in order to accurately model the important details on large-space scales (few $\mathrm{m}$ ) and long-time scales (few $\mu$ s). In addition, large densities and magnetic fields can lead to prohibitively small time steps in traditional, explicit PIC simulations because of the need to resolve fast phenomena occurring at the electron plasma frequency, $\omega_{p, e}$, and the electron cyclotron frequency, $\omega_{c, e}$, which can both exceed $10^{11} \mathrm{rad} \mathrm{s}^{-1}$ in some situations. Implicit techniques in PIC codes typically allow the under-resolution of one of the frequencies, but not both simultaneously. Therefore, even modest magnetic field 
values can hinder the use of a larger time step which would allow PIC simulations to compete with other reduced numerical models in terms of required computational resources.

The macroscopic warm-fluid description for charged particle beam dynamics is a continuum approximation, derived by evaluating velocity moments of the Vlasov equation. Closure of the system of equations in the present analysis is achieved by assuming that heat flow is negligible between the beam and its surroundings and by ignoring the higher-order moments. The resulting approximate equations form a closed description of the bulk fluid properties, the dynamics of which are reversible as well as isentropic.

The adoption of a warm-fluid model, which forms a coupled, nonlinear partial differential equation (PDE) system without the use of macroparticles, can yield numerical results which are less noisy, provide more stability, are computationally efficient, and can conserve energy to predetermined levels of precision that are not generally accessible in second-order particle-pushing routines commonly used in PIC codes. In the beam frame, the nonrelativistic warm-fluid equations in the longitudinal direction for the line density $\lambda(z, t)$, flow velocity $u_{z}(z, t)$, and pressure $p_{z}(z, t)$ are given by $[24,26]$

$$
\begin{gathered}
\frac{\partial}{\partial t} \lambda(z, t)+\frac{\partial}{\partial z}\left[\lambda(z, t) u_{z}(z, t)\right]=0, \\
\frac{\partial}{\partial t} u_{z}(z, t)+u_{z}(z, t) \frac{\partial}{\partial z} u_{z}(z, t)-\frac{q}{m} E_{z}^{\mathrm{tilt}}(z, t) \\
+f(z, t) \frac{q^{2} g}{4 \pi \epsilon_{0} m} \frac{\partial}{\partial z} \lambda(z, t)+\frac{1}{\lambda(z, t)} \frac{r_{b}^{2}}{m} \frac{\partial}{\partial z} p_{z}(z, t)=0,
\end{gathered}
$$

$$
\frac{\partial}{\partial t} p_{z}(z, t)+u_{z}(z, t) \frac{\partial}{\partial z} p_{z}(z, t)+3 p_{z} \frac{\partial}{\partial z} u_{z}(z, t)=0,
$$

where $p_{z}(z, t)=n(z, t) T_{z}(z, t), T_{z}(z, t)$ is the effective longitudinal temperature, and zero heat flux $\left(q_{z}=0\right)$ is assumed. The above equations correspond to the continuity equation, momentum equation, and pressure equation, respectively, for a macroscopic fluid element of beam ions with charge $q$ and mass $m$. The beam ions are subjected to an external electric field $E_{z}^{\text {tilt }}(z, t)$, which corresponds to the space- and time-dependent induced electric field the beam encounters in the (finite) acceleration gap of the induction module in the longitudinal compression experiments.

The perveance term is proportional to $\partial \lambda(z, t) / \partial z$ in the momentum equation and is associated with the space charge of the ion beam. The coefficient $f(z, t)$ of that term is a function describing the degree of charge neutralization by the background plasma where $f=0$ and $f=1$ correspond to fully neutralized and fully unneutralized ion beam charge, respectively, and the $g$-factor occurring in that term is a model $[26,28]$ for the longitudinal selfelectric field of the ion beam, with $g=2 \ln \left(r_{w} / r_{b}\right)$, where $r_{w}$ and $r_{b}$ are the wall radius and beam radius, respectively. Pressure effects are important to include near the focal plane of the compressed charge bunch because of chromatic aberration of the longitudinal focal plane, a consequence of considering finite beam temperature effects. For an ideal velocity tilt, beam particles with small deviations from the mean axial velocity will be longitudinally focused to different focal planes, causing decreased compression factors and increased pulse durations at those planes. For example, beam ions with velocity $u_{z}+u_{\mathrm{th}, z}$ will be moving too quickly for a given velocity tilt and will longitudinally focus later in time (and space) than those moving with velocity $u_{z}$; the opposite is true for beam ions with velocity $u_{z}-u_{\mathrm{th}, z}$.

The goal of using the warm-fluid equations as a numerical model for comparison to measurements from neutralized drift compression experiments is to employ the experimental voltage waveform used by the induction module in order to apply a nonideal velocity tilt to an ion beam which has nonzero longitudinal thermal spread and subsequently propagates through a partially neutralizing background plasma, corresponding to a nonzero value of $f(z, t)$. A plentiful supply of electrons is desired in order to effectively neutralize the beam's space-charge, implying $n_{\text {beam }} \ll n_{\text {plasma }}$ everywhere "downstream" of the applied tilt, thereby ensuring the propagation of beam currents well beyond the space-charge limit of unneutralized beams. However, the beam density can approach values of the plasma density depending on the particular experimental profiles provided for $n_{\text {plasma }}(\mathbf{r}, t)$, especially near the simultaneous transverse and longitudinal focal plane where the beam's current density rises more rapidly due to the small spot size achieved by means of transverse compression. For this reason, it can be especially important to include the perveance and pressure terms in Eqs. (1)-(3) in the numerical framework.

There exist other reasons for implementing a fast and accurate numerical model based on the warm-fluid equations. First, such a model allows for the lifting of restrictions on uniformity, symmetry, and solution-type (e.g., self-similar) which have often been assumed in earlier studies. Second, a fluid model allows the determination of whether the observed features of the beam variables such as velocity and current are due to kinetic effects. Third, a nonparticle-based numerical method allows the efficient and noiseless study of how deviations from ideal voltage waveforms used by the induction module affect the evolution of the profiles (line density, velocity, and pressure).

The numerical method of lines $[29,30]$ is used in order to solve the nonlinear warm-fluid PDE system after the problem has been well posed with initial conditions and bound- 
ary values [31]. In general, the method of lines is efficient and can take advantage of a wide range of numerical integration techniques $[32,33]$. There are two primary steps to the method of lines: first, the spatial dimension is discretized and, second, the semidiscrete temporal problem is integrated as a system of ordinary differential equations (ODEs) $[34,35]$. In the present analysis, the discretization is carried out to fourth order using centered finite differences [36], although finite element and finite volume methods can alternatively be employed. The ODEs are numerically integrated in time using a high-order predictor-corrector Adams method [37] based on the Maclaurin series with backward differences [38].

In order to describe the NDCX device and investigate the physics of neutralized drift compression in the laboratory, a theoretical model needs to incorporate the actual spatial and temporal dependence of the induced electric field found in the finite acceleration gap of the induction module. The acceleration gap begins at the wall radius of $3.8 \mathrm{~cm}$, is $3 \mathrm{~cm}$ long in the axial direction, and is symmetric about the azimuth. The top image in Fig. 2 illustrates the connection between the effective voltage drop across the acceleration gap, which is located from $Z=-19 \mathrm{~cm}$ to $-22 \mathrm{~cm}$ at radii larger than the wall radius, and the resultant induced electric field found at radii less than the wall radius (where the beam encounters the applied force). The bottom image in Fig. 2 shows the longitudinal spatial dependence of the axial electric field at the $r=0$ axis, i.e., the form of $E_{z}^{\text {tilt }}(z)$ used in the momentum equation of the fluid model. The axial coordinate used in the laboratory frame for NDCX models extends from $Z=-30 \mathrm{~cm}$ to

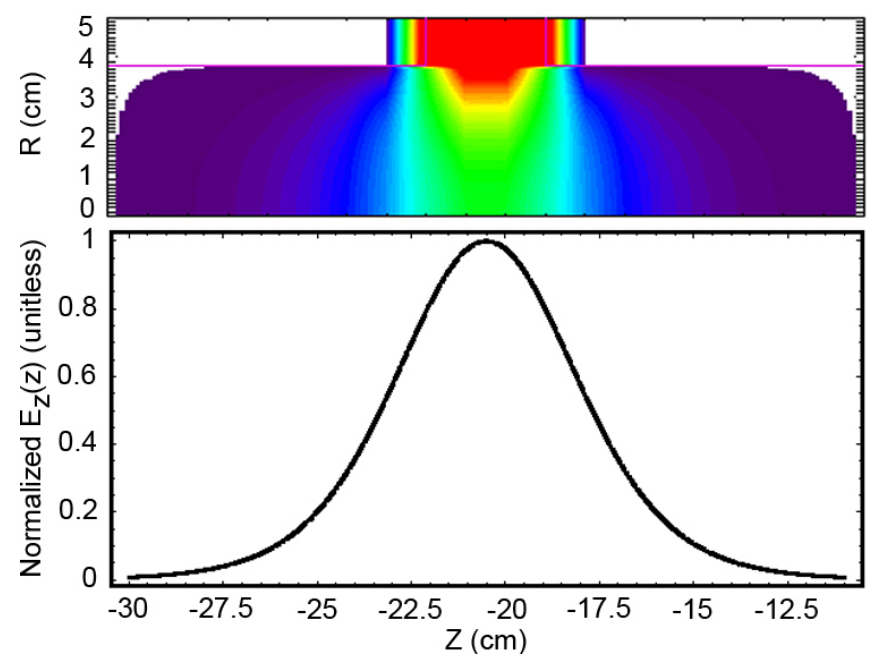

FIG. 2. (Color) In models describing NDCX, the axial coordinate is measured in the laboratory frame from $Z=-30 \mathrm{~cm}$ to $Z=+120 \mathrm{~cm}$. Top: Snapshot (in time) of the $E_{z}^{\text {tilt }}(r, z)$ in the acceleration gap, calculated from the geometry of the currently existing induction module on NDCX. Bottom: Slice of $E_{z}^{\text {tilt }}(r=$ $0, z)$ used in the fluid model to describe the longitudinal spatial dependence of the axial electric field.
$Z=+120 \mathrm{~cm}$, and the background neutralizing plasma exists from $Z=0 \mathrm{~cm}$ to $Z=+120 \mathrm{~cm}$.

Figure 3 highlights the difference between a typical experimental voltage waveform used in the induction module and an ideal waveform which would result in the imposition of a perfect linear velocity tilt on an ion beam with zero temperature across a thin $\left(\Delta z \ll r_{\text {wall }}\right)$ acceleration gap. The experimental waveform plotted in Fig. 3 is used to calculate the temporal dependence of the axial electric field at the $r=0$ axis, $E_{z}^{\text {tilt }}(t)$, used in the momentum equation of the fluid model.

In order to compare the code based on the fluid model to a large-scale PIC simulation, the LSP PIC code $[39,40]$ was used. The use of the LSP simulation code is one method for simulating an ion beam pulse propagating through a background plasma. LSP is an advanced 3D electromagnetic PIC code, available commercially and designed for complex, large-scale plasma simulations which can be run in multiple coordinate systems and geometries. The LSP code has been utilized in order to realistically predict the evolution of the ion beam as it acquires a velocity tilt and drifts through the plasma to the focal plane.

The same initial parameters used in LSP simulations were used in the warm-fluid code. In the LSP simulation, a $300 \mathrm{keV} K^{+}$beam with a $2 \mathrm{~cm}$ radius was initialized upstream of the induction module (at $Z=-30 \mathrm{~cm}$ ), with a $0.2 \mathrm{eV}$ temperature in both the transverse and longitudinal directions, and injected into the NDCX geometry in the $+\hat{z}$ direction. The experimental voltage waveform (shown in Fig. 3) was used in the simulation and resulted in the application of a $25 \%$ velocity tilt (defined as $\Delta u_{z} / u_{z}^{\text {initial})}$ to the beam over the acceleration gap before the beam

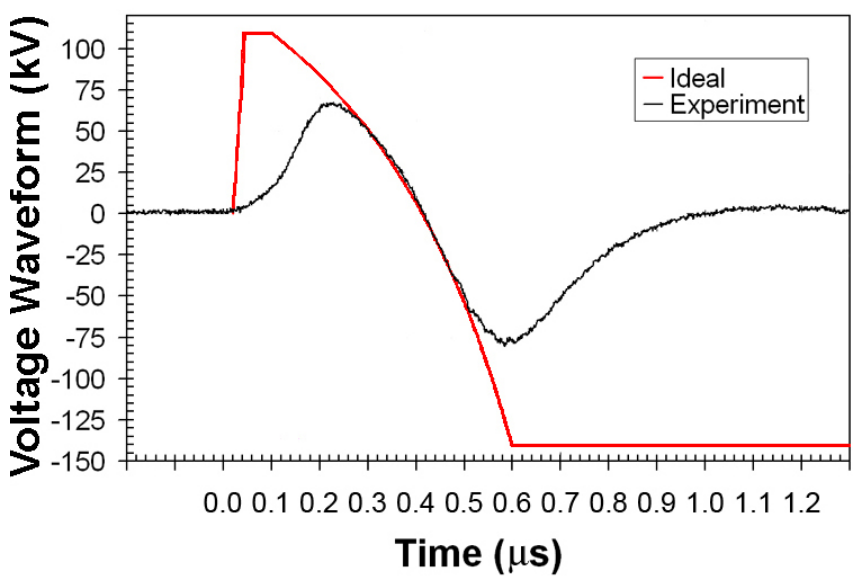

FIG. 3. (Color) Comparison between the experimental voltage waveform (black) used in the induction module in order to apply the $E_{z}^{\text {tilt }}(t)$ in the acceleration gap and the ideal voltage waveform (red) which would impose an ideal linear velocity tilt on the drifting beam (from 0.1 to $0.6 \mu \mathrm{s}$ ). The experimental voltage waveform is used for the temporal dependence of the axial electric field, $E_{z}^{\text {tilt }}(t)$, in the fluid model. 
drifted through a plasma column filled with background plasma of $10^{11} \mathrm{~cm}^{-3}$ density and $3 \mathrm{eV}$ temperature (from $Z=0 \mathrm{~cm}$ to $Z=+120 \mathrm{~cm}$ ). The LSP simulation demonstrated that the plasma almost completely neutralized the charge and current of the ion beam. A longitudinal compression factor of 50 (defined as $I^{\text {max }} / I^{\text {initial }}$ ) with a fullwidth, half-maximum pulse duration of $3.2 \mathrm{~ns}$ was achieved for the given beam parameters. The longitudinal focal plane occurred near $Z=+110 \mathrm{~cm}$, approximately $1.3 \mathrm{~m}$ downstream of the acceleration gap.

Many observed features in the beam current profiles from the PIC simulation of neutralized drift compression are recovered using the fluid model. Since NDCX experiments have concentrated on longitudinal compression, and not simultaneously focusing the beam in the transverse direction, the beam density remains at least an order of magnitude smaller than the background plasma density $\left(10^{11} \mathrm{~cm}^{-3}\right)$ at all locations in the device; also, LSP simulations showed good ( $>95 \%$ ) neutralization of the beam's space charge by the background plasma, in agreement with theory when $n_{\text {beam }} \ll n_{\text {plasma }}[9,10,16]$. Therefore, the $f(z, t)$ coefficient of the perveance term in the momentum equation of the fluid model is taken to be small $(<0.05)$ in the presence of plasma from $Z=0 \mathrm{~cm}$ to $Z=+120 \mathrm{~cm}$; the $f(z, t)$ coefficient is set to unity in the region of the acceleration gap, where no plasma is present. When only longitudinal compression takes place (without simultaneous transverse focusing), the perveance term in the momentum equation is not dominant for these beam parameters and little difference in the compression physics can be found between a completely neutralized beam $(f=$ 0 in the plasma region) and an almost completely neutralized beam $(f<0.05$ in the plasma region). However, when simultaneous transverse and longitudinal compression takes place, the perveance becomes the critical term for determining the achievable amount of compression for a charge bunch, due to the strong dependence of the current density on the beam radius, and substantial differences are found between the completely neutralized and almost completely neutralized cases. These are topics for future research.

The current is calculated in the fluid model from the product $\lambda(z, t) u_{z}(z, t)$ and transformed to the laboratory frame in order to compare with the LSP diagnostics which measure the beam current at a specified plane in laboratory coordinates. Since the current through a plane is the transverse integral of the current density through that plane, a direct comparison can be made between the PIC simulations and the warm-fluid model calculations in the longitudinal direction. Figure 4 illustrates the longitudinal compression results (in the laboratory frame) obtained in the PIC simulations and the corresponding profiles calculated using the fluid model. The definition of the compression ratio is $I(t) / I_{\text {initial }}$ (the initial beam current in NDCX can vary between 10 and $40 \mathrm{~mA}$ ). There is excellent

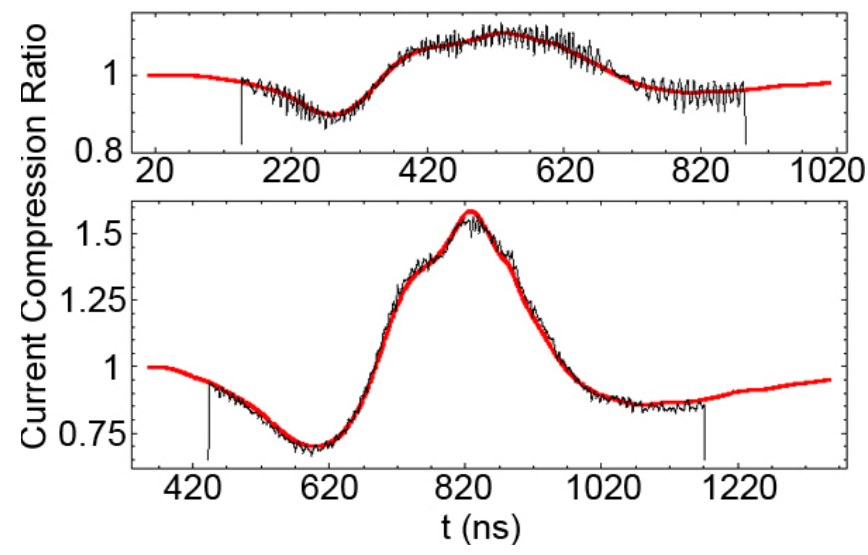

FIG. 4. (Color) Longitudinal compression ratio $I(t) / I_{\text {initial }}$ in the laboratory frame. Top: PIC simulation (black) and fluid model (red) at $Z=-8 \mathrm{~cm}$, just beyond the acceleration gap. Bottom: PIC simulation (black) and fluid model (red) at $Z=+29 \mathrm{~cm}$.

agreement between the two results, indicating that the various features (e.g., asymmetric dips and bumps) in the current profile of the ion beam are entirely due to the nonideal, experimental voltage waveform used to apply the velocity tilt to the beam over the actual acceleration gap. Such features cannot be observed in models which do not use a finite acceleration gap and the experimental voltage waveform.

A comparison of the longitudinal current compression profiles, at various axial locations in the beam frame from $Z=-8 \mathrm{~cm}$ to $Z=92 \mathrm{~cm}$, predicted by the two models is shown in Fig. 5. The envelope of the compression ratio seen at $Z=0 \mathrm{~cm}$ in the beam frame, which is the center of the charge bunch and where the decelerated head meets the accelerated "tail," is plotted as a dashed line. Such an envelope should touch each individual current profile at the location of peak compression compared to the rest of the beam: the center of the beam, moving at the average beam velocity, would experience a buildup of density at that location as the beam drifts and compresses through a neutralizing background plasma. It is evident from Fig. 5 that the fluid results are in very good quantitative agreement with the kinetic PIC simulations; hardly any discrepancy is evident, especially early in time.

An interesting consequence of the experimental velocity-tilting process is that the $E_{z}^{\text {tilt }}(z, t)$ in the acceleration gap acts not only as a means for pulse-shaping the velocity of the ion beam, but it also pulse shapes the line density. Specifically, the line density profile in the beam frame, which is initially constant (or a step function), is transformed by this particular tilt application process into a parabolic line density profile. Figure 6 depicts the line density and axial velocity profiles calculated in the beam frame from the fluid model after the tilt has been applied to the beam, and the pertinent portion of the voltage waveform of the induction module has passed $(t=830 \mathrm{~ns}$, see Fig. 3). At $t=830 \mathrm{~ns}$, the center of the beam in the 


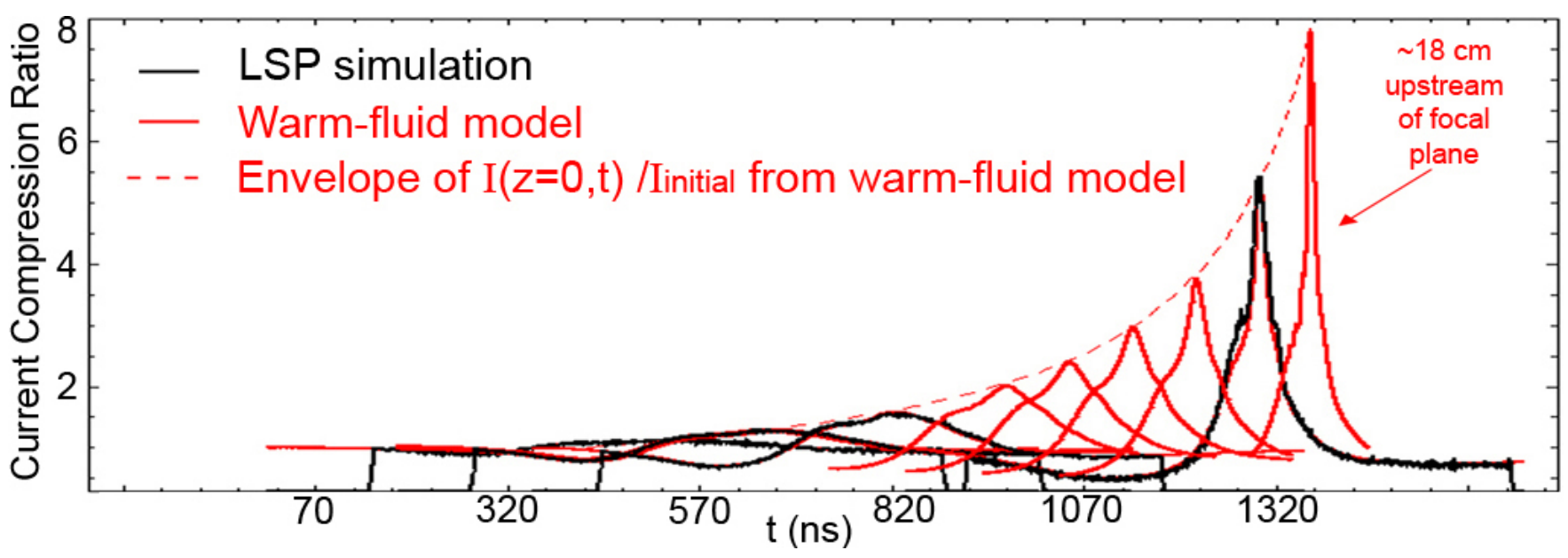

FIG. 5. (Color) Longitudinal compression ratio $I(t) / I_{\text {initial }}$ in the laboratory frame obtained from the PIC simulation (black) and fluid model (red) at multiple axial locations from $Z=-8 \mathrm{~cm}$ to $Z=+92 \mathrm{~cm}$.

laboratory frame is located at $Z=+29 \mathrm{~cm}$, about $81 \mathrm{~cm}$ upstream from the longitudinal focal plane. The quantities have been normalized to the initial line density and the initial beam frame axial velocity (an arbitrary shift of +1 has been applied to the normalized velocity profile since the initial beam frame axial velocity is zero). Note that the center of the tilted beam is unchanged, as it must be for a linear velocity tilt applied by a voltage waveform which symmetrically switches polarity. The parabola $2(1-$ $z^{2} / 36$ ), where $z$ is in $\mathrm{cm}$, is plotted as a dashed line in the center of the beam frame in order to exhibit the fact that the portion of the beam that was tilted very well (corresponding to the linear region in the velocity profile) has had its constant line density profile transformed into a parabolic line density profile. The pressure profile (not shown) has been transformed, in the region of the linear velocity tilt, into a double-parabolic profile. Information on such

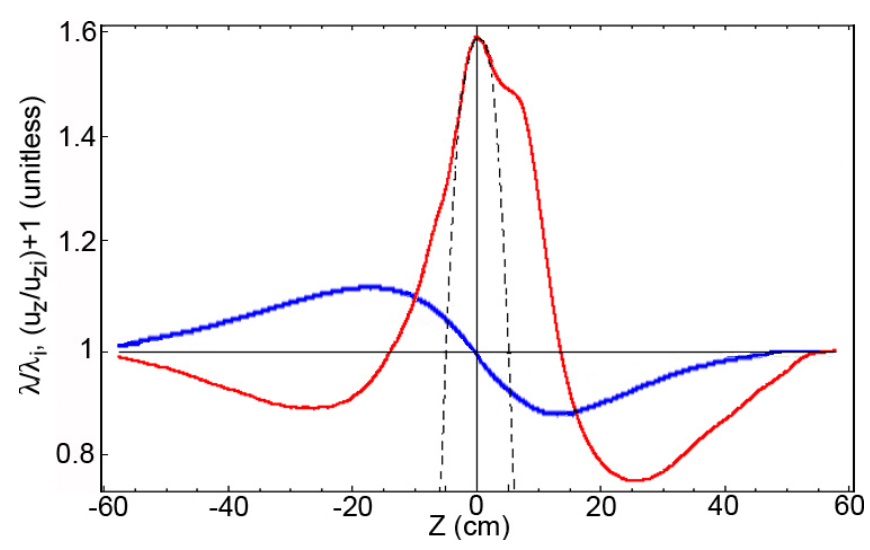

FIG. 6. (Color) Normalized line density (red) and axial velocity (blue) in the beam frame after most of the velocity tilt has been applied ( $t=830 \mathrm{~ns}$ ). The center of the beam is located at $Z=$ $+29 \mathrm{~cm}$ in the laboratory frame at this instant of time. The dashed line is the parabola $2\left(1-z^{2} / 36\right)$, where $z$ is in $\mathrm{cm}$. transformations of the beam profiles is valuable, since a parabolic line density profile and double-parabolic pressure profile with a linear velocity tilt is a self-similar drift compression solution in the longitudinal direction of the warm-fluid model, as well as a particular solution to the Vlasov equation in 1D when complete neutralization of the beam is assumed. Drift compression solutions that satisfy a self-similar symmetry condition can be used in models in order to transversely focus the entire beam pulse onto the same focal spot on the target [26]. Solutions obeying selfsimilarity are required for this procedure because, for each slice in a bunched beam, a drift compression and finalfocus lattice design can transversely focus all the different slices onto the same focal spot only if the current $\lambda(z, t) u_{z}(z, t)$ and emittance [related to $p_{z}(z, t)$ ] of all the slices depend on the longitudinal coordinate in the same manner.

The family of self-similar profiles under discussion is

$$
\begin{gathered}
\lambda(z, t)=\lambda_{b}(t)\left[1-z^{2} / z_{b}(t)^{2}\right], \\
u_{z}(z, t)=-u_{b}(t) z / z_{b}(t), \\
p_{z}(z, t)=p_{z, b}(t)\left[1-z^{2} / z_{b}(t)^{2}\right],
\end{gathered}
$$

where $z_{b}(t), \lambda_{b}(t), u_{b}(t)$, and $p_{z, b}(t)$ are time-dependent scale factors, measured relative to the center of the beam. Note that the length of the ion beam pulse, in the beam frame, which is affected by the linear velocity tilt is $L_{b}(t=$ $830 \mathrm{~ns})=2 z_{b}(t=830 \mathrm{~ns})=12 \mathrm{~cm}$ in Fig. 6 , which corresponds to about $100 \mathrm{~ns}$ of the $300 \mathrm{keV} K^{+}$ion beam undergoing the desired drift compression (at $t=830 \mathrm{~ns}$ ). Following Qin et al. [26], it can be shown that the functions of time $\lambda_{b}(t), u_{b}(t)$, and $p_{z, b}(t)$ are invariants of the nonclassical point symmetry group transformation. Substituting the invariants into the warm-fluid equations will 
result in the loss of dependence on the axial coordinate $z$ and recovery of the longitudinal envelope equation for those invariants.

The inadequacy in treatment of the full distribution function, $f_{b}(\mathbf{x}, \mathbf{v}, t)$, is a weak point in the fluid description of beams. A kinetic description of the beam propagating through background plasma evolves a distribution function through space and time without many of the assumptions and constraints imposed by the fluid model. Another significant drawback of a macroscopic one-fluid model in this particular case is its inability to support multiple-valued functions. For instance, some nonideal tilts will cause the $u_{z}(z)$ velocity dependence of the beam to become multiple valued when slow particles, which were decelerated too much, meet fast particles, which were accelerated too much. Such a multiple-valued axial velocity function can occur anywhere from a few to dozens of $\mathrm{cm}$ upstream of the longitudinal focus, especially when the experimental voltage waveform is above the ideal waveform early in time and below the ideal waveform late in time (see Fig. 3). Even when the applied waveform has an ideal shape, the one-fluid solution cannot be propagated to the longitudinal focus: the axial velocity profile will become close to vertical as the beam reaches peak longitudinal compression and the focus will necessarily be composed of many fluid elements with different flow velocities but with similar positions. However, formalisms based on the Vlasov equation can be used to investigate the propagation of a longitudinally compressed beam up to its focus.

\section{KINETIC MODEL}

A kinetic model based on the Vlasov equation has recently been developed for the propagation of an intense ion charge bunch which undergoes simultaneous transverse and longitudinal focusing in a background plasma, assuming complete charge and current neutralization of the ion charge bunch [22]. Thus, the self-field forces of the ion beam are neglected in the model. Under such circumstances, the Vlasov equation can be solved exactly for a wide range of initial distribution functions, $f_{b}(\mathbf{x}, \mathbf{v}, 0)$, yielding dynamically compressing solutions [22]. The configuration of a device such as NDCX, which performs the neutralized drift compression of intense ion beams, can be investigated numerically using the framework of this kinetic model and comparisons can be made to PIC simulations [18] as well as to fast Faraday cup measurements of the longitudinally compressed ion beam [19].

Many choices of beam equilibria are possible for use in such a kinetic model of neutralized drift compression. It was shown in Sec. II that the acceleration gap and timedependent voltage waveform of the induction module can transform, in the beam frame, a constant line density profile into a parabolic line density profile in the region where a linear velocity tilt has been imposed on the drifting ion beam. This observation is relevant to the task of simul- taneous transverse and longitudinal focusing of an ion beam because the line density, axial velocity, and pressure profiles under discussion comprise a self-similar drift compression solution and can be used in models in order to transversely focus the entire beam pulse onto the same focal spot on the target [26]. Therefore, for an ion beam which has already acquired a linear velocity tilt and whose line density profile has been transformed into one with parabolic shape, we consider as an example the distribution function

$$
f_{b}\left(W_{\perp}, W_{z}\right)=A \sqrt{\left(1-W_{z}\right)} \exp \left(-W_{\perp}\right)
$$

where $A$ is a constant, and $0 \leq W_{z}<1$. The constants of the motion, $W_{\perp}$ and $W_{z}$, are defined as

$$
\begin{aligned}
W_{\perp}= & \frac{x^{2}+y^{2}}{r_{b}^{2}(t)}+\frac{r_{b}^{2}(t)}{r_{b, 0}^{2} v_{\mathrm{th}, \perp}^{2}}\left[\left(v_{x}-\frac{x}{r_{b}(t)} \frac{d r_{b}(t)}{d t}\right)^{2}\right. \\
+ & \left.\left(v_{y}-\frac{y}{r_{b}(t)} \frac{d r_{b}(t)}{d t}\right)^{2}\right] \\
& W_{z}=\frac{z^{2}}{z_{b}^{2}(t)}+\frac{z_{b}^{2}(t)}{z_{b, 0}^{2} v_{t h, z}^{2}}\left(v_{z}-\frac{z}{z_{b}(t)} \frac{d z_{b}(t)}{d t}\right)^{2} .
\end{aligned}
$$

The quantities $r_{b}(t)$ and $z_{b}(t)$ are related to the rms transverse and axial dimensions of the charge bunch, and in the absence of an applied focusing magnetic field solve the effective envelope equations [22]

$$
\begin{aligned}
\frac{d^{2} r_{b}(t)}{d t^{2}} & =\frac{r_{b, 0}^{2} v_{\mathrm{th}, \perp}^{2}}{r_{b}^{3}(t)} \\
\frac{d^{2} z_{b}(t)}{d t^{2}} & =\frac{z_{b, 0}^{2} v_{\mathrm{th}, z}^{2}}{z_{b}^{3}(t)}
\end{aligned}
$$

where the constants $r_{b, 0}^{2} v_{\mathrm{th}, \perp}^{2}$ and $z_{b, 0}^{2} v_{\mathrm{th}, z}^{2}$ are scaled transverse and longitudinal emittances. The constituent constants are the initial radius at the midplane, perpendicular thermal velocity, bunch length $\left(L_{b}=2 z_{b}\right)$, and parallel thermal velocity of the beam ions. Then, the dynamical evolution of the number density of the charge bunch in the beam frame is given by

$$
\begin{aligned}
n_{b}(r, z, t)= & N_{b} \frac{\theta\left\{\left[z_{b}(t)+z\right]\left[z_{b}(t)-z\right]\right\}}{\pi^{3 / 2} r_{b, 0}^{2} z_{b, 0}}\left(\frac{r_{b, 0}^{2} z_{b, 0}}{r_{b}^{2}(t) z_{b}(t)}\right) \\
& \times\left[1-\left(\frac{z}{z_{b}(t)}\right)^{2}\right] \exp \left[-r^{2} / r_{b}^{2}(t)\right],
\end{aligned}
$$

where $\theta$ is the Heaviside step function, and $N_{b}=$ $\int d^{3} x \int d^{3} v f_{b}$ is a constant equal to the number of particles in the charge bunch. In this model, the number density is maximum at $(r, z, t)=(0,0, t)$, which is the center of the beam pulse in the beam frame. Note that, for this particular 
choice of $f_{b}\left(W_{\perp}, W_{z}\right)$, the line density profile is parabolic, the longitudinal pressure profile is double parabolic, and the axial velocity profile is linearly dependent on $z$, i.e.,

$$
v_{z}(z, t)=\frac{z}{z_{b}(t)} \frac{d z_{b}(t)}{d t}
$$

with an initial negative tilt, $\left[d z_{b}(t) / d t\right]_{t=0}<0$, ensuring that the beam longitudinally compresses from its initial bunch length $L_{b, 0}=2 z_{b, 0}$ at $t \equiv t_{\text {kinetic }}=0$.

The kinetic model has been solved numerically after being given appropriate initial conditions which closely represent the experimental parameters measured in NDCX. Since this model initializes a parabolic line density profile which already has a linear velocity tilt applied to it, the numerical solution of the beam's evolution begins with the center of the beam located at the center of the acceleration gap at $t_{\text {kinetic }}=0$. However, a relative temporal offset is involved because the warm-fluid model and LSP simulations include the finite amount of time required to apply the $E_{z}^{\text {tilt }}$ to the passing ion beam in the acceleration gap and transform the line density from a constant to parabolic profile. The $300 \mathrm{keV}, K^{+}$charge bunch was initialized

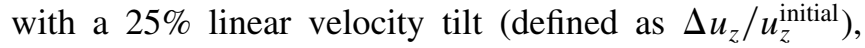
temperature of $0.2 \mathrm{eV}$ in both the transverse and longitudinal directions, $r_{b, 0}=2 \mathrm{~cm}$, and pulse half-length of $z_{b, 0}=9.6 \mathrm{~cm}$. A pulse length of $19.2 \mathrm{~cm}$ corresponds to a pulse duration of $160 \mathrm{~ns}$ for the velocity involved here (the ion beam's pulse length is often quoted in terms of its duration, which is the beam length divided by the beam velocity).

The transverse and axial dimensions of the charge bunch, $r_{b}(t)$ and $z_{b}(t)$, are both decreasing functions of time (until the focal plane is reached) for the initial conditions which specify compression in both directions:

$$
\begin{gathered}
r_{b}(t=0)=r_{b, 0}, \\
{\left[d r_{b}(t) / d t\right]_{t=0}=-r_{b, 0} / L_{\mathrm{drift}},} \\
z_{b}(t=0)=z_{b, 0}, \\
{\left[d z_{b}(t) / d t\right]_{t=0}=-z_{b, 0} / L_{\mathrm{drift}},}
\end{gathered}
$$

where $L_{\text {drift }}$ is the drift length required for the velocitytilted ion beam to come to longitudinal focus $\left(L_{\text {drift }} \sim\right.$ $1.3 \mathrm{~m}$, here). Once the effective envelope equations, Eqs. (10) and (11), are solved for $r_{b}(t)$ and $z_{b}(t)$, the dynamical evolution of the number density of the charge bunch in the beam frame can be solved according to Eq. (12). For the case where the ion beam's charge and current are completely neutralized, the peak beam density expected at the focal plane gives insight into the density requirements for the neutralizing background plasma near that location. Recent research indicates that beam com- pression will begin to stagnate due to defocusing spacecharge forces when $n_{\text {beam }}>n_{\text {plasma }}$, which can occur near a simultaneous focal plane even when large plasma densities are employed, as the beam density increases quadratically with decreasing beam radius. However, the present paper is only concerned with longitudinal compression in a neutralizing background plasma. Experiment, theory, and simulation of simultaneous radial and longitudinal focusing of an ion beam will be addressed in future investigations.

The solutions obtained from the kinetic model, as used in this paper, can be directly compared to those from the other models beginning at axial and temporal locations sufficiently far removed so as not to be affected by the acceleration gap region. For example, consider an axial location of $Z=+29 \mathrm{~cm}$ in the laboratory frame, since, by that location, the fluid model and the LSP simulations clearly exhibit that a major portion of their solutions (near the center of the beam) have a parabolic line density profile with linear velocity tilt (see Fig. 6). The initialization described in Eqs. (14)-(17) assures that the kinetic solution coincides at the appropriate time with the parabolic line density profile (shown as a dashed line in Fig. 6) assembled downstream of the acceleration gap by the actual $E_{z}^{\text {tilt }}(z, t)$ used in the fluid model and LSP simulations. Recall that, for the kinetic model, the center of the charge bunch $(z=0 \mathrm{~cm})$ is initialized at the location of the center of the acceleration gap with a linear velocity tilt already applied to it. The self-similar parabolic line density solution is then longitudinally compressed by the linear velocity tilt to a pulse length of $z_{b}(t)=6 \mathrm{~cm}$, which corresponds to a duration of $100 \mathrm{~ns}$, after an elapsed time of $t_{\text {kinetic }}=410 \mathrm{~ns}$; the warm-fluid model assembled the same solution at the center of the beam (see Fig. 6) after an elapsed time of $t=830 \mathrm{~ns}$. Both solutions coincide at the axial coordinate $Z=+29 \mathrm{~cm}$ in the laboratory frame. Evidently, the temporal offset between the two models for the mentioned parameters is $420 \mathrm{~ns}$, which is also the location in time of the sign change of the voltage waveform (and polarity switch of the axial electric field) of the induction module (see Fig. 3). This observation is true because the center of the beam is unaffected by the applied velocity tilt and exits the acceleration gap traveling at the initial beam velocity, which is enforced by the kinetic model $\left[v_{z}(z=0, t)=0\right.$ for all time $]$.

Figure 7 shows the comparison between the longitudinal compression ratios [defined as $I(t) / I_{\text {initial }}$ ] predicted by the kinetic model and those predicted by LSP simulation for a number of axial locations throughout the drift region, including the longitudinal focal plane. The kinetic model results are transformed to the laboratory frame and calculated from $I(z, t)=2 \pi e_{b} \int d r r n_{b}(r, z, t) v_{z}(z, t)$ in order to compare to the laboratory frame current probes from the PIC code. The axial flow velocity in the kinetic model is defined in Eq. (13). A current compression ratio of $50 \times$ the 


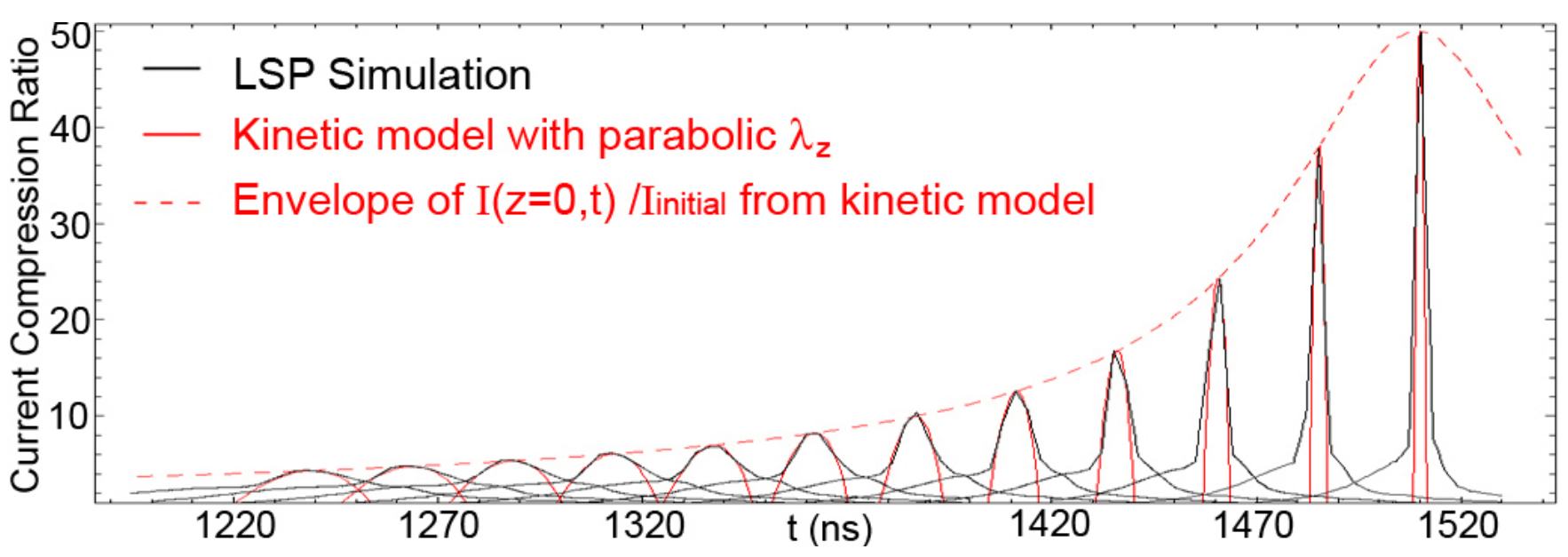

FIG. 7. (Color) Longitudinal compression ratio $I(t) / I_{\text {initial }}$ in the laboratory frame obtained from the PIC simulation (black) and kinetic model (red) at multiple axial locations near the focal plane from $Z=+77 \mathrm{~cm}$ to $Z=+110 \mathrm{~cm}$.

initial current with a pulse duration of $\sim 3.2 \mathrm{~ns}$ is predicted by both models. The initial current of the NDCX $K^{+}$beam was $25 \mathrm{~mA}$ and is determined by the choice of constant $\left(N_{b}\right)$ in Eq. (12). The peak ion beam density expected at the focal plane is a function of both $r_{b}(t)$ and $z_{b}(t)$; for a longitudinal current compression of 50 and a focal spot radius of $r_{b}^{\min }=1 \mathrm{~cm}$, the peak beam density achieved at the focal plane is approximately $2 \times 10^{10} \mathrm{~cm}^{-3}$. This result indicates that the assumption that the plasma completely neutralized the ion beam space charge is reliable, as typical plasma densities in the NDCX device are $5 \times$ $10^{10} \mathrm{~cm}^{-3}$ to $1 \times 10^{11} \mathrm{~cm}^{-3}$ and theory predicts good neutralization under these circumstances.

The parabolic line density profile with a linear velocity tilt accounts for the highly compressed region found near the center of the beam. Evidently, the parts of the beam which were not tilted well end up forming a pedestal of current around the peak of current in each profile near the longitudinal focal plane. Figure 8 highlights the amount of deviation between the experimental and ideal voltage waveforms, which is the cause of pedestal formation. By charge conservation arguments, since the peaks in the current profiles near focus are due to the parts of the beam which did participate in the region of linear velocity tilt, the formation of pedestals correspondingly reduces the total amount of current compression achieved at the beam's center. Therefore, pedestals around the current peaks are undesirable and should be minimized, especially those in front of the main pulse since unwanted preheat of the target may occur.

It is important to note that the kinetic formalism, as used in this paper, only applies to the center of the beam which is tilted well, since the charge bunch was initialized with a linear velocity tilt and parabolic line density profile. In fact, Figs. 6 and 7 indicate that the majority of the beam affected by the experimental voltage waveform does not participate in neutralized drift compression within the region of the linear velocity tilt. Therefore, to understand dynamics of the parts of the beam that interact with the nonlinear part of the voltage waveform, and hence become part of the pedestal, the space- and time-dependent electric force found in the acceleration gap needs to be included in the model. Such an external force can be accounted for in the kinetic model as long as the self-forces of the beam can be neglected in the acceleration gap and a dense background plasma is provided in the drift region, since the model requires complete neutralization of the beam's charge and current. Provided an initial distribution function, $f_{b}(\mathbf{x}, \mathbf{v}, 0)$, the kinetic model [22] permits an exact determination of the beam's distribution function for all later times, $f_{b}(\mathbf{x}, \mathbf{v}, t)$, which can be used to investigate the detailed dynamic focusing of the charge bunch, in both the longitudinal and transverse directions, for the case of complete charge and current neutralization.

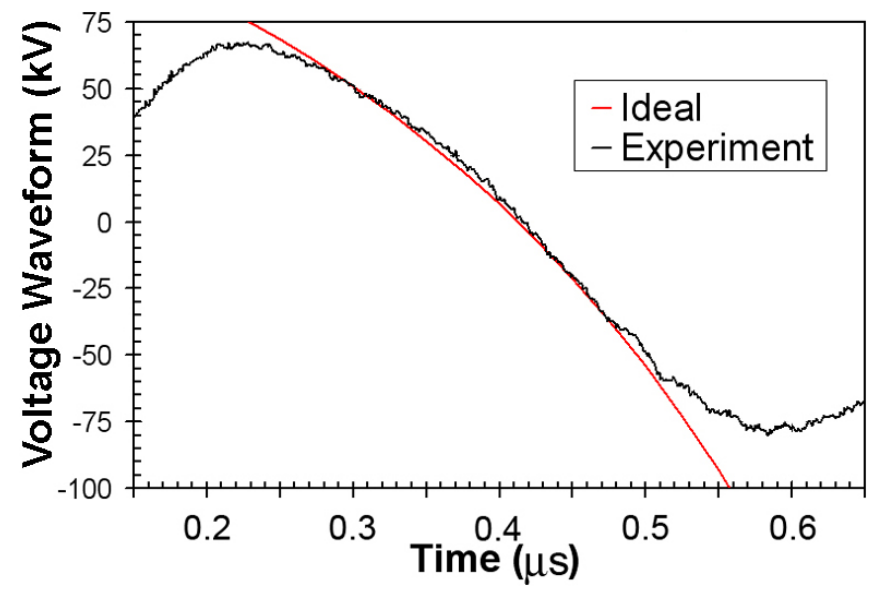

FIG. 8. (Color) Comparison between the experimental voltage waveform (black) and the ideal voltage waveform (red) that would impose an ideal linear velocity tilt on the drifting beam. The axes have been reduced in scale relative to Fig. 3 in order to more clearly illustrate the differences between the two. 


\section{HYBRID FLUID-VLASOV MODEL}

The warm-fluid and kinetic models presented in the previous sections each have advantages and disadvantages. The main advantage (disadvantage) of the fluid (kinetic) model is its ability (inability) to correctly anticipate the nonideal effects of an experimental voltage waveform's influence on the line density, axial velocity, and pressure profiles of the charge bunch as it undergoes neutralized drift compression. The main disadvantage (advantage) of the one-fluid (kinetic) code is its inability (ability) to propagate such a solution to the longitudinal focal plane when the flow velocity becomes multiple valued. A hybrid combination of the two theoretical models is found to provide a satisfactory formalism for describing longitudinal drift compression in the NDCX device from injection to focus.

Given the fluid variables $\lambda(z, t), u_{z}(z, t)$, and $p_{z}(z, t)$, a distribution function $f\left(z, v_{z}, t\right)$ can be constructed, which allows the propagation, by means of the 1D Vlasov equation, of the current profiles $I(z, t)$ of a charge bunch to the longitudinal focus. Such a construction neglects the selfforces of the ion beam, so the assumption in using the hybrid model is the complete neutralization of the ion beam's charge and current by the background plasma throughout the drift region. This assumption holds true for recent NDCX experiments, which have concentrated on longitudinal focusing without simultaneous transverse focusing to small spot size, where very good ( $>95 \%$ ) charge neutralization has been verified. Finally, the hybrid approach is most accurate in the regime where imperfections in the voltage waveform dominate the expected compression ratios and final pulse durations (this is currently the operating regime of NDCX). In the other limit, when voltage waveforms are nearly ideal, beam temperature effects give rise to chromatic aberration of the longitudinal focal plane and partial neutralization effects by the plasma (if present) give rise to defocusing self-forces (since the ion beam propagates beyond the traditional space-charge limit). Both effects are expected to play a role in the determination of the upper limit of achievable current density compression when experimental voltage waveform imperfections no longer dominate.

The 1D Vlasov equation (neglecting space charge and external forces) in the longitudinal direction is $\partial f / \partial t+$ $v_{z} \partial f / \partial z=0$ and the general solution is a function of two variables, $\xi=z-v_{z}\left(t-t_{0}\right)$ and $v_{z}$, i.e.,

$$
f\left(z, v_{z}, t\right)=f\left[z-v_{z}\left(t-t_{0}\right), v_{z}, 0\right],
$$

where

$$
\xi=z-v_{z}\left(\xi, t_{0}\right)\left(t-t_{0}\right)
$$

and

$$
v_{z}=\frac{z-\xi}{t-t_{0}}
$$

Assuming an initial Gaussian distribution with thermal velocity $v_{\mathrm{th}, z}$, the current profile as a function of space and time for specified "initial" $\lambda(z, t)$ and $u_{z}(z, t)$ fluid variables is given by

$$
\begin{aligned}
I(z, t)= & \frac{1}{\left(t-t_{0}\right)^{2}} \int \frac{d \xi}{\sqrt{2 \pi} v_{\mathrm{th}, z}} \lambda\left(\xi, t_{0}\right)(z-\xi) \\
& \times \exp \left(-\frac{\left[z-\xi-v_{z}\left(\xi, t_{0}\right)\left(t-t_{0}\right)\right]^{2}}{2\left(t-t_{0}\right)^{2} v_{\mathrm{th}, z}^{2}}\right) .
\end{aligned}
$$

Here, $\lambda\left(z=\xi, t=t_{0}\right)$ and $v_{z}\left(\xi, t_{0}\right)=u_{z}\left(z=\xi, t=t_{0}\right)$ are evaluated from the warm-fluid model at a time $t=t_{0}$ after the voltage waveform of the induction module has returned to zero ( $t \sim 1 \mu \mathrm{s}$ was chosen here), corresponding to the required condition of no external forces. Effectively,

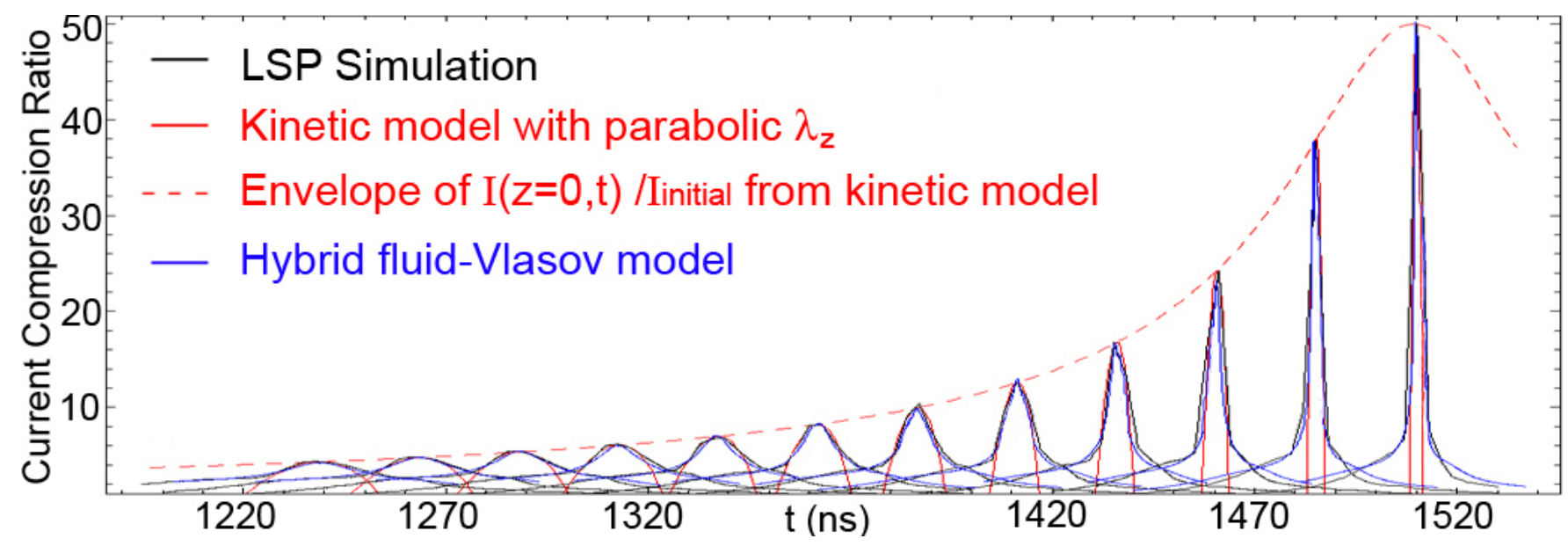

FIG. 9. (Color) Longitudinal compression ratio $I(t) / I_{\text {initial }}$ in the laboratory frame obtained from the PIC simulation (black), kinetic model (red), and hybrid model (blue) at multiple axial locations near the focal plane from $Z=+77 \mathrm{~cm}$ to $Z=+110 \mathrm{~cm}$. 
the adoption of the hybrid model combines the advantages of the fluid and kinetic formalisms by propagating the warm-fluid solution to longitudinal focus by treating it as an initial condition in the 1D Vlasov equation.

The hybrid model solutions compared to those from the kinetic model and PIC simulation are shown in Fig. 9. Very good agreement is obtained between the full PIC simulation and the hybrid fluid-Vlasov treatment. Evidently, the formation of the pedestals in current due to beam interaction with the nonlinear part of the voltage waveform is described very well by the hybrid model.

\section{COMPARISON BETWEEN THEORETICAL MODELS AND EXPERIMENT}

In order to compress the ion beam in the longitudinal direction in the NDCX device, the time-dependent voltage waveform of the induction module imposes a velocity tilt on the passing ion beam. The beam drifts through a plasma column until a focal plane in current density is created between 1 and 2 meters downstream of the gap (the length of the plasma column can be changed from 1 to 2 meters in the experiments). The exact location of the longitudinal focal plane of the beam is determined by the slope of the compressing part $(d V / d t<0)$ of the voltage waveform, as well as by the velocity of the injected ion beam. A high degree of space-charge neutralization by the background plasma is required in order to compress intense ion beams to current densities of interest for heavy ion fusion and high energy density physics applications.

In recent NDCX experiments, the timing of the voltage waveform is set to act upon the middle $2 \mu \mathrm{s}$ of a $6 \mu$ s-long beam. A fast Faraday cup which measures the absolute beam current in the presence of plasma as a function of time [19] has been designed, fabricated, and installed and is located at the far end of the plasma region such that the longitudinal focal plane coincides with the plane of the collector plate. Experiments have confirmed that the collector only measures a signal proportional to the ion beam current, without the negative influence of the other charged particle species in the system. The fast Faraday cup measurements have corroborated optical measurements made by a scintillator and fast phototube arrangement [41]; however, only the fast Faraday cup diagnostic can measure the actual ion beam current in the presence of plasma.

Figure 10 plots the peak longitudinal compression factor obtained in recent measurements on NDCX using the fast Faraday cup [19] and provides a comparison with results from LSP simulations, the kinetic model, and the hybrid model. The experimental result is an average of the four best shots measured with the fast Faraday cup at the focal plane, which range in compression factors (defined as $\left.I_{b}^{\max } / I_{b}^{\text {initial }}\right)$ from 55 to 65 . The compressed ion beam signal has been normalized to the initial beam current (without an applied tilt) and expanded around the location of peak compression in order to show the error bars. The

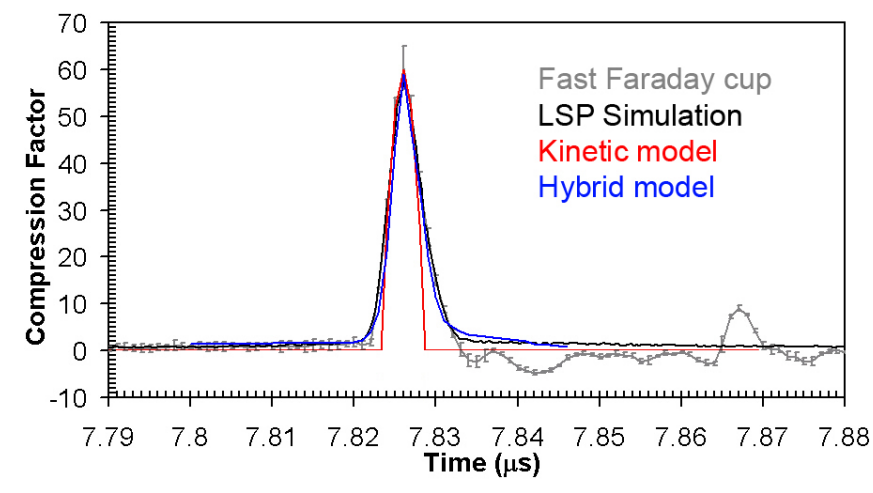

FIG. 10. (Color) Comparison of longitudinal current compression at the focal plane between the experimental measurement using a fast Faraday cup (gray with error bars), LSP simulation (black), kinetic model (red), and hybrid model (blue).

error bars plotted for the experiment are the 95\% confidence intervals in the mean of the four shots. Very good agreement between experiment and the hybrid model is found for this case which achieved a current compression factor of $60 \times$ with a pulse duration of about $4 \mathrm{~ns}$. The voltage waveforms used in these experiments were able to more closely approximate the ideal waveform over a wider interval of time and, therefore, compressed more of the ion beam pulse relative to the waveform shown in Figs. 3 and 8.

The hybrid model and the PIC simulation very accurately reproduce the measured amount of longitudinal compression as well as the pulse duration. The kinetic model accurately reproduced the region of peak compression in the central portion of the beam but did not include the formation of the current pedestal due to the imperfectly tilted beam, as discussed earlier. It is believed that the shortest achievable pulse durations of the compressed ion beams in the recent NDCX experiments have been limited by the experimental voltage waveform; as mentioned, the experimental waveform is expected to dominate the compression dynamics because of the nonlinear deviations from the ideal waveform which give rise to current pedestal formation at the cost of lower total compression and longer pulse durations. If the beam had an ideal slope to its velocity tilt, then chromatic aberration due to longitudinal temperature effects and partial neutralization effects (if present) are expected to dominate the minimum achievable pulse duration, as mentioned in Sec. IV. Other interesting experimental results and simulations pertaining to NDCX, especially as they relate to the need for precise control over the slope of the induction module waveform which applies the head-to-tail velocity tilt to the drifting ion beam, have been published [42].

\section{CONCLUSIONS}

This paper has demonstrated that reduced theoretical models can be effective in describing the longitudinal 
compression of an intense charge bunch in neutralizing background plasma. A warm-fluid model has been analyzed (Sec. II) as a tractable computational tool for investigating nonideal effects associated with the longitudinal compression of intense ion beams to high current densities. The fluid model demonstrated that the inclusion of the actual acceleration gap geometry and experimental voltage waveform of the induction module is important in order to make realistic comparisons to experiments with neutralized drift compression. The main observed features in the beam profiles were recovered with the fluid model, indicating that their origin lies in the finite nature of the acceleration gap and do not require kinetic explanations. It was observed that the applied $E_{z}^{\text {tilt }}(z, t)$ in the acceleration gap acts as a means for pulse shaping not only the axial velocity profile, but also the line density profile in the beam frame. A favorable consequence of the pulse-shaping ability of the acceleration gap is that ion beam profiles with a linear velocity tilt, parabolic line density profile, and double-parabolic pressure profile belong to a family of self-similar drift compression solutions which can be used to transversely focus the charge bunch to the same focal plane for warm dense matter and heavy ion fusion applications.

A kinetic model based on the Vlasov equation has also been employed (Sec. III) in order to understand the physics of the central portion of the beam which acquires a linear velocity tilt with a parabolic line density profile. The kinetic model was also used to show that, when a beam has an experimental voltage waveform applied to it, the peak in the current profile is a result of the fact that only the central portion of the beam which was tilted well (given a linear axial velocity profile) contributes effectively to the main compressed pulse. Significant portions of the charge bunch do not reside in the linearly compressing part of the velocity profile because of deviations between the experimental and ideal voltage waveforms of the induction module. In order to study how those regions form a pedestal of current around the central peak, thereby decreasing the total amount of compression and increasing the pulse duration achieved at the focal plane, a hybrid model was developed (Sec. IV) by propagating a 1D Vlasov solution for $I(z, t)$, which was initialized with profiles calculated from the warm-fluid model. Such a hybrid implementation combines the main advantages of both methods. The successful comparison between the experimental measurement from the NDCX device and the various theoretical models presented in this paper is very encouraging.

\section{ACKNOWLEDGMENTS}

The authors would like to acknowledge useful discussions with Dr. Igor Kaganovich, Dr. Hong Qin, Dr. Prabir Roy, Dr. Peter Seidl, Dr. Edward Startsev, Dr. Dale Welch, Dr. Simon Yu, Mahmood Miah, and Justin Stimatze. This research was supported by the U.S. Department of Energy under the auspices of the Heavy Ion Fusion Science Virtual National Laboratory.

[1] B. G. Logan, R. O. Bangerter, D. A. Callahan, M. Tabak, M. Roth, L. J. Perkins, and G. Gaporaso, Fusion Sci. Technol. 49, 399 (2006).

[2] B. Y. Sharkov, N. N. Alexeev, M. M. Basko, M.D. Churazov, D. G. Koshkarev, S. A. Medin, Y. N. Orlov, and V. M. Suslin, Nucl. Fusion 45, S291 (2005).

[3] T. Kikuchi, T. Someya, S. Kawata, M. Nakajima, K. Horioka, and T. Katayama, Nucl. Instrum. Methods Phys. Res., Sect. A 558, 122 (2006).

[4] M. Reiser, Theory and Design of Charged Particle Beams (John Wiley \& Sons, Inc., New York, 1994).

[5] R. B. Miller, An Introduction to the Physics of Intense Charged Particle Beams (Plenum Press, New York, 1994).

[6] R.C. Davidson and H. Qin, Physics of Intense Charged Particle Beams in High Energy Accelerators (World Scientific, Singapore, 2001).

[7] D. A. Callahan, Fusion Eng. Des. 32-33, 441 (1996).

[8] B. G. Logan and D. A. Callahan, Nucl. Instrum. Methods Phys. Res., Sect. A 415, 468 (1998).

[9] I. D. Kaganovich, G. Shvets, E. Startsev, and R.C. Davidson, Phys. Plasmas 8, 4180 (2001).

[10] D. V. Rose, D. R. Welch, B. V. Oliver, R. E. Clark, W. M. Sharp, and A. Friedman, Nucl. Instrum. Methods Phys. Res., Sect. A 464, 299 (2001).

[11] E. Henestroza, S. Eylon, P. K. Roy, S. S. Yu, A. Anders, F. M. Bieniosek, W. G. Greenway, B. G. Logan, R. A. MacGill, D. B. Shuman, D. L. Vanecek, W.L. Waldron, W. M. Sharp, T.L. Houck, R.C. Davidson, P.C. Efthimion, E. P. Gilson, A. B. Sefkow, D. R. Welch, D. V. Rose, and C.L. Olson, Phys. Rev. ST Accel. Beams 7, 083501 (2004).

[12] P. K. Roy, S. S. Yu, S. Eylon, E. Henestroza, A. Anders, E.P. Gilson, F.M. Bieniosek, W. G. Greenway, B. G. Logan, W. L. Waldron, D. B. Shuman, D. L. Vanecek, D. R. Welch, D. V. Rose, R.C. Davidson, P.C. Efthimion, I. D. Kaganovich, A. B. Sefkow, and W. M. Sharp, Nucl. Instrum. Methods Phys. Res., Sect. A 544, 255 (2005).

[13] P. K. Roy, S.S. Yu, S. Eylon, E. Henestroza, A. Anders, F. M. Bieniosek, W. G. Greenway, B. G. Logan, W. L. Waldron, D. L. Vanecek, D. R. Welch, D. V. Rose, R. C. Davidson, P. C. Efthimion, E. P. Gilson, A. B. Sefkow, and W. M. Sharp, Phys. Plasmas 11, 2890 (2004).

[14] D. R. Welch, D. V. Rose, B. V. Oliver, and R. E. Clark, Nucl. Instrum. Methods Phys. Res., Sect. A 464, 134 (2001).

[15] C. H. Thoma, D. R. Welch, S. S. Yu, E. Henestroza, P. K. Roy, S. Eylon, and E. P. Gilson, Phys. Plasmas 12, 043102 (2005).

[16] D. R. Welch, D. V. Rose, S. S. Yu, J. J. Barnard, and C. L. Olson, Nucl. Instrum. Methods Phys. Res., Sect. A 544, 236 (2005).

[17] N. Christofilos, R. Hester, W. Lamb, D. Reagan, W. Sherwood, and R. Wright, Rev. Sci. Instrum. 35, 886 (1964). 
[18] A. B. Sefkow, R.C. Davidson, P.C. Efthimion, E. P. Gilson, S. S. Yu, P. K. Roy, S. Eylon, F. M. Bieniosek, E. Henestroza, J. W. Kwan, J.E. Coleman, W. L. Waldron, W. G. Greenway, D. L. Vanecek, and D. R. Welch, Proceedings of the 2005 Particle Accelerator Conference (2005), p. 3765, http://ieeexplore.ieee.org/xpls/abs_all. jsp?arnumber $=1591610$.

[19] A. B. Sefkow, R.C. Davidson, P.C. Efthimion, E.P. Gilson, S. S. Yu, P. K. Roy, F. M. Bieniosek, J.E. Coleman, S. Eylon, W. G. Greenway, E. Henestroza, J. W. Kwan, D. L. Vanecek, W. L. Waldron, and D. R. Welch, Phys. Rev. ST Accel. Beams 9, 052801 (2006).

[20] H. Qin, R. C. Davidson, J. J. Barnard, and E. P. Lee, Phys. Rev. ST Accel. Beams 7, 104201 (2004).

[21] W. M. Sharp, J. J. Barnard, D. P. Grote, C. M. Celata, and S. S. Yu, Nucl. Instrum. Methods Phys. Res., Sect. A 544, 398 (2005).

[22] R. C. Davidson and H. Qin, Phys. Rev. ST Accel. Beams 8, 064201 (2005).

[23] R.C. Davidson, Physics of Nonneutral Plasmas (World Scientific, Singapore, 2001).

[24] H. Qin and R. C. Davidson, Phys. Rev. ST Accel. Beams 5, 034401 (2002).

[25] H. Qin and R.C. Davidson, Laser Part. Beams 20, 565 (2002).

[26] H. Qin, R. C. Davidson, J. J. Barnard, and E. P. Lee, Nucl. Instrum. Methods Phys. Res., Sect. A 544, 255 (2005).

[27] C. K. Birdsall and A.B. Langdon, Plasma Physics via Computer Simulation (McGraw-Hill Book Company, New York, 1985).

[28] R. C. Davidson and E. A. Startsev, Phys. Rev. ST Accel. Beams 7, 024401 (2004).

[29] W. Schiesser, The Numerical Method of Lines (Academic Press, San Diego, 1991).

[30] A. Quarteroni and A. Valli, Numerical Approximation of Partial Differential Equations (Springer-Verlag, Berlin, 1994).
[31] R. Richtmeyer and K. Morton, Difference Methods for Initial Value Problems (Krieger Publishing Company, Florida, 1994).

[32] J.D. Lambert, Numerical Methods for Ordinary Differential Equations (John Wiley and Sons, Chichester, 1987).

[33] L.F. Shampine, Numerical Solution of Ordinary Differential Equations (Chapman and Hall, New York, 1994).

[34] L. Shampine and M. Gordon, Computer Solutions of Ordinary Differential Equations (W.H. Freeman, San Francisco, 1975).

[35] U. Ascher and L. Petzold, Computer Methods for Ordinary Differential Equations and DifferentialAlgebraic Equations (SIAM Press, Philadelphia, 1998).

[36] A. Mitchell and D. Griffiths, The Finite Difference Method in Partial Differential Equations (John Wiley and Sons, New York, 1980).

[37] F. Bashforth and J.C. Adams, Theories of Capillary Action (Cambridge University Press, London, 1883).

[38] W. H. Beyer, CRC Standard Mathematical Tables (CRC Press, Boca Raton, FL, 1987).

[39] LSP is a software product of ATK Mission Research, Albuquerque, NM 87110.

[40] T. P. Hughes, S. S. Yu, and R. E. Clark, Phys. Rev. ST Accel. Beams 2, 110401 (1999).

[41] F. M. Bieniosek, S. Eylon, A. Faltens, A. Friedman, J. W. Kwan, M. A. Leitner, A. W. Molvik, L. Prost, P. K. Roy, P. A. Seidel, and G. Westenskow, Nucl. Instrum. Methods Phys. Res., Sect. A 544, 268 (2005).

[42] P. K. Roy, S.S. Yu, E. Henestroza, A. Anders, F. M. Bieniosek, J. Coleman, S. Eylon, W. G. Greenway, M. Leitner, B. G. Logan, W. L. Waldron, D. R. Welch, C. Thoma, A. B. Sefkow, E.P. Gilson, P. C. Efthimion, and R.C. Davidson, Phys. Rev. Lett. 95, 234801 (2005). 